\title{
Theoretical analysis of highly spin-polarized transport in the iron nitride $\mathrm{Fe}_{4} \mathrm{~N}$
}

\author{
Satoshi Kokado, ${ }^{1} *$ Nobuhisa Fujima, ${ }^{1}$ Kikuo Harigaya, ${ }^{2}$ Hisashi Shimizu, ${ }^{3}$ and Akimasa Sakuma ${ }^{4}$ \\ ${ }^{1}$ Faculty of Engineering, Shizuoka University, Hamamatsu 432-8561, Japan \\ ${ }^{2}$ Nanotechnology Research Institute, AIST, Tsukuba 305-8568, Japan \\ ${ }^{3}$ Faculty of Science, Shinshu University, Matsumoto 390-8621, Japan \\ ${ }^{4}$ Graduate School of Engineering, Tohoku University, Sendai 980-8579, Japan
}

(Received 12 December 2005; revised manuscript received 17 April 2006; published 17 May 2006)

\begin{abstract}
In order to propose a ferromagnet exhibiting highly spin-polarized transport, we theoretically analyzed the spin polarization ratio of the conductivity of the bulk $\mathrm{Fe}_{4} \mathrm{~N}$ with a perovskite-type structure, in which $\mathrm{N}$ is located at the body center position of the fcc-Fe. The spin polarization ratio is defined by $P=\left(\sigma_{\uparrow}-\sigma_{\downarrow}\right) /\left(\sigma_{\uparrow}\right.$ $+\sigma_{\downarrow}$ ), with $\sigma_{\uparrow(\downarrow)}$ being the conductivity at zero temperature of the up spin (down spin). The conductivity is obtained by using the Kubo formula and the Slater-Koster tight binding model, where parameters are determined from the least-square fitting of the dispersion curves by the tight binding model to those by the first principles calculation. In the vicinity of the Fermi energy, $|P|$ takes almost 1.0, indicating a perfectly spinpolarized transport. In addition, by comparing $\mathrm{Fe}_{4} \mathrm{~N}$ to fcc-Fe $\left(\mathrm{Fe}_{4} \mathrm{~N}_{0}\right)$ in the ferromagnetic state with the equilibrium lattice constant of $\mathrm{Fe}_{4} \mathrm{~N}$, it is shown that the nonmagnetic atom $\mathrm{N}$ plays an important role in increasing $|P|$.
\end{abstract}

DOI: 10.1103/PhysRevB.73.172410

PACS number(s): 72.25.Ba

Recently, highly efficient spin-electronics devices operating at room temperature have been extensively developed for applications to the magnetic memory and the magnetic sensor. A typical device has ferromagnetic tunnel junctions consisting of a ferromagnetic electrode (FME)/insulator/FME, which exhibits a large magnetoresistance (MR) effect. ${ }^{1-7}$ The efficiency of the MR effect is often defined by the MR ratio $=\left(R_{\mathrm{AP}}-R_{\mathrm{P}}\right) / R_{\mathrm{AP}}$, with $R_{\mathrm{P}}$ and $R_{\mathrm{AP}}$ being the resistance of the parallel and antiparallel magnetization configurations of FMEs, respectively. Experimentally, regarding junctions with an electrode of a half-metallic ferromagnet, $\mathrm{Co}_{2} \mathrm{Cr}_{0.6} \mathrm{Fe}_{0.4} \mathrm{Al} / \mathrm{Al}-\mathrm{O} / \mathrm{CoFe} / \mathrm{NiFe} / \mathrm{IrMn} / \mathrm{Ta}$ junctions exhibited a MR ratio of $16 \%$ at room temperature, ${ }^{1}$ and $\mathrm{Co}_{2} \mathrm{MnSi} / \mathrm{Al}-\mathrm{O} / \mathrm{Co}_{75} \mathrm{Fe}_{25}$ had a $\mathrm{MR}$ ratio of $70 \%$ at room temperature. ${ }^{2}$ Regarding junctions of electrodes with usual ferromagnets, single-crystal $\mathrm{Fe}(001) / \mathrm{MgO}(001) / \mathrm{Fe}(001)$ junctions exhibited MR ratios of $88 \%$ (Ref. 3) and $180 \%$ (Ref. 4) at room temperature. Furthermore, $\mathrm{CoFeB} / \mathrm{MgO} / \mathrm{CoFeB}$ junctions achieved MR ratios of $260 \%$ (Ref. 5) and 355\% (Ref. 6) (the world's highest value) at room temperature, although the crystal structure of $\mathrm{CoFeB}$ and the role of the light element $\mathrm{B}$ on the spin-polarized transport have not been clarified yet. Generally, the MR ratio becomes large by increasing the spin polarization of the conduction electron in the FME. In the future, a FME with more highly spin-polarized electrons, which would result in larger MR ratios, will be strongly desired from the viewpoint of the development of highly efficient MR devices.

Towards a proposal of such a FME, we extracted an idea to obtain electrodes exhibiting the highly spin-polarized transport at room temperature. We found that ferromagnets consisting of magnetic elements and light elements, such as $\mathrm{CoFeB}$, might be very useful as the electrodes.

We consider $\mathrm{Fe}$ as a representative magnetic element in this idea. We define the spin polarization (SP) ratio about the density of states (DOS) of the bulk system, $P_{\mathrm{DOS}}(E)$, as

$$
P_{\mathrm{DOS}}(E)=\frac{D_{\uparrow}(E)-D_{\downarrow}(E)}{D_{\uparrow}(E)+D_{\downarrow}(E)},
$$

with $D_{s}(E)$ being the DOS of spin $s(=\uparrow$ or $\downarrow)$ for the energy $E$. Then, $\left|P_{\mathrm{DOS}}\left(E_{\mathrm{F}}\right)\right|$ at the Fermi energy $E_{\mathrm{F}}$ of fcc-Fe in the ferromagnetic state is evaluated to be about 0.7 [see Fig. 2(c)], which is about 2.3 times as large as that of bcc-Fe, ${ }^{8}$ where the most stable state of bcc-Fe (fcc-Fe) is ferromagnetic (not ferromagnetic ${ }^{9}$ ). This may indicate that the highly spin-polarized transport is realized for materials closely related to fcc-Fe. The conductivity, including the velocity of electrons, will give an answer to the realizability of such transport.

We focus on a ferromagnet containing fcc-Fe and a light element $\mathrm{Fe}_{4} \mathrm{~N}$ with a perovskite-type structure, ${ }^{10-15}$ in which $\mathrm{N}$ is located at the body center position of fcc-Fe. This ferromagnet has a Curie temperature of $761 \mathrm{~K} .{ }^{12}$ It should be noted that studies on the spin-polarized transport of $\mathrm{Fe}_{4} \mathrm{~N}$ have scarcely been performed so far, although other properties have been experimentally ${ }^{10-12}$ and theoretically ${ }^{13-15}$ investigated. We are particularly interested in not only the SP ratio on the transport of $\mathrm{Fe}_{4} \mathrm{~N}$ but also the role of the light (or nonmagnetic) element $\mathrm{N}$ on the transport.

In this paper, we analyzed the SP ratio of the conductivity of $\mathrm{Fe}_{4} \mathrm{~N}$ in order to elucidate the spin-polarized transport. The conductivity was obtained for each spin and each orbital of the bulk system using the first principles (FP) calculation and the tight binding (TB) model calculation. Consequently, we found that $\mathrm{Fe}_{4} \mathrm{~N}$ exhibits an extremely highly spinpolarized transport and that $\mathrm{N}$ plays an important role in the transport.

A calculation method is introduced to obtain the conductivity and the SP ratio. The method is a combination of (i) the FP calculation, ${ }^{16}$ (ii) the TB model, ${ }^{17}$ and (iii) the Kubo formula. ${ }^{18}$ Details of each are given below.

(i) The FP calculations are performed by the Vienna $a b$ 
initio Simulation Package (VASP) code $^{16}$ based on the spinpolarized density functional theory, where we employ the generalized gradient approximation of Perdew and Wang ${ }^{19}$ and ultrasoft pseudopotentials to describe the core electrons. The cutoff energy for the plane wave basis is $237.51 \mathrm{eV}$ for $\mathrm{Fe}$ and $348.10 \mathrm{eV}$ for $\mathrm{Fe}_{4} \mathrm{~N}$, and the Monkhorst-Pack set ${ }^{20}$ of $8 \times 8 \times 8 \mathbf{~ k}$ points is used.

(ii) The Slater-Koster TB mode $1^{17}$ is used while taking into account the $3 d, 4 s$, and $4 p$ orbitals for $\mathrm{Fe}$ and the $2 s$ and $2 p$ orbitals for $\mathrm{N}$ as well as interactions up to the thirdnearest-neighbor atoms. The Slater-Koster parameters of potential energies and transfer integrals ${ }^{17}$ are here determined from the least-square fitting of the dispersion curves by the TB model to those by the FP calculation at the equilibrium lattice constant. The fitting is done in energy regions from the lowest energy to $E_{\mathrm{F}}+5 \mathrm{eV}$ for bcc-Fe and fcc-Fe and from the lowest energy to $E_{\mathrm{F}}+2 \mathrm{eV}$ for $\mathrm{Fe}_{4} \mathrm{~N}$. The number of parameters is 34 for bcc-Fe and fcc-Fe and 87 for $\mathrm{Fe}_{4} \mathrm{~N}$.

(iii) The Kubo formula ${ }^{18}$ and the Slater-Koster TB model with the determined parameters are used to calculate the conductivity at zero temperature. In this calculation, we utilize the theory given by Tsymbal et al. ${ }^{21}$ The total conductivity of $\operatorname{spin} s(=\uparrow$ or $\downarrow), \sigma_{s}(E)$, is written by

$$
\sigma_{s}(E)=\sum_{i} \sigma_{i, s}(E),
$$

where $\sigma_{i, s}(E)$ is the conductivity of orbital $i(=4 s, 4 p, 3 d$ orbitals, and so on) and spin $s$. This $\sigma_{i, s}(E)$ includes the veloc-
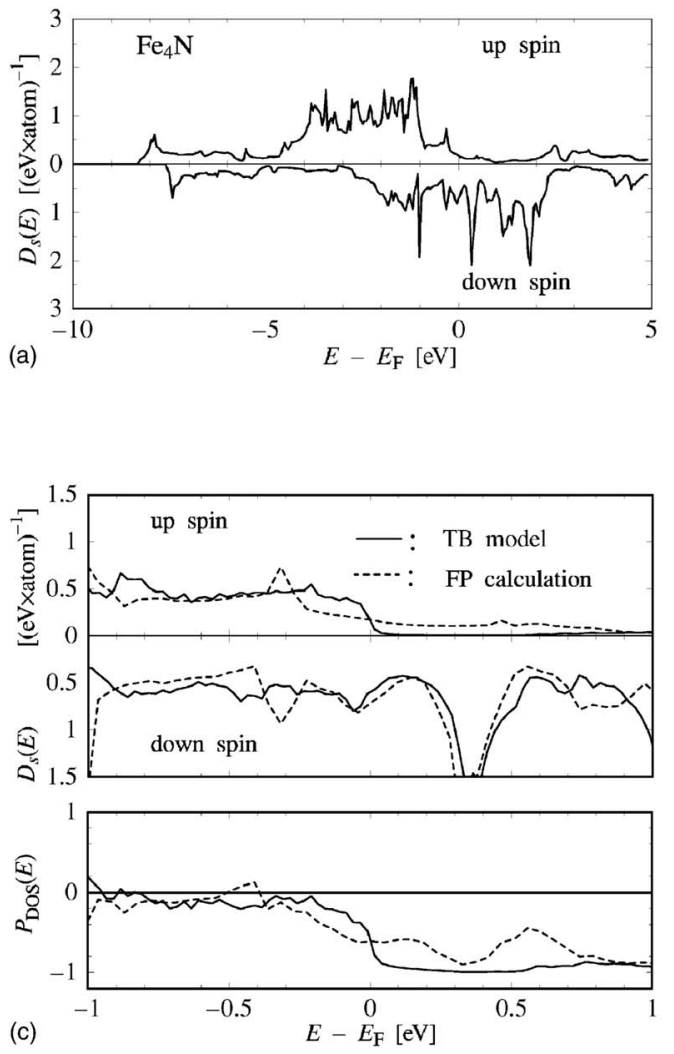

ity of electrons and the Green's function. The Green's function has a single parameter $\gamma$ in the second-order self-energy due to the weak electron-impurity interaction, where $\gamma$ characterizes the degree of electron-impurity scattering. In detail, $\gamma$ is related to the lifetime of the electron of orbital $i$ and spin $s, \tau_{i, s}$, via $1 / \tau_{i, s}=(2 \pi / \hbar) \gamma^{2} D_{i, s}(E)$, with $D_{i, s}(E)$ being the partial DOS for $i$ and $s .^{21}$ At present, $\gamma$ is set to be $0.5 \mathrm{eV}$, which was previously chosen so as to reproduce the resistivity of copper. ${ }^{21} \mathrm{We}$ also use the diagonal approximation for the self-energy, in which the scattering of electrons due to impurities is allowed for the same energy levels but forbidden between different energy levels. ${ }^{21}$ The SP ratio is then defined by

$$
P(E)=\frac{\sigma_{\uparrow}(E)-\sigma_{\downarrow}(E)}{\sigma_{\uparrow}(E)+\sigma_{\downarrow}(E)} .
$$

We first compare the results of bcc-Fe by the present method with the previous ones. The equilibrium lattice constant obtained by using the FP calculation is estimated to be $2.84 \AA$ with an error of about $1 \%$ for the experimental value of $2.87 \AA .{ }^{22}$ The total conductivity at $E_{\mathrm{F}}$ of the down spin $\sigma_{\downarrow}\left(E_{\mathrm{F}}\right)$ is larger than $\sigma_{\uparrow}\left(E_{\mathrm{F}}\right)$. The SP ratio at $E_{\mathrm{F}}, P\left(E_{\mathrm{F}}\right)$, therefore takes a negative value, -0.20 , and it qualitatively agrees with the previous result of about $-0.26,{ }^{21}$ which was obtained by a similar method combined with the FP calculation within the local density approximation.

In the following, we investigate the equilibrium lattice
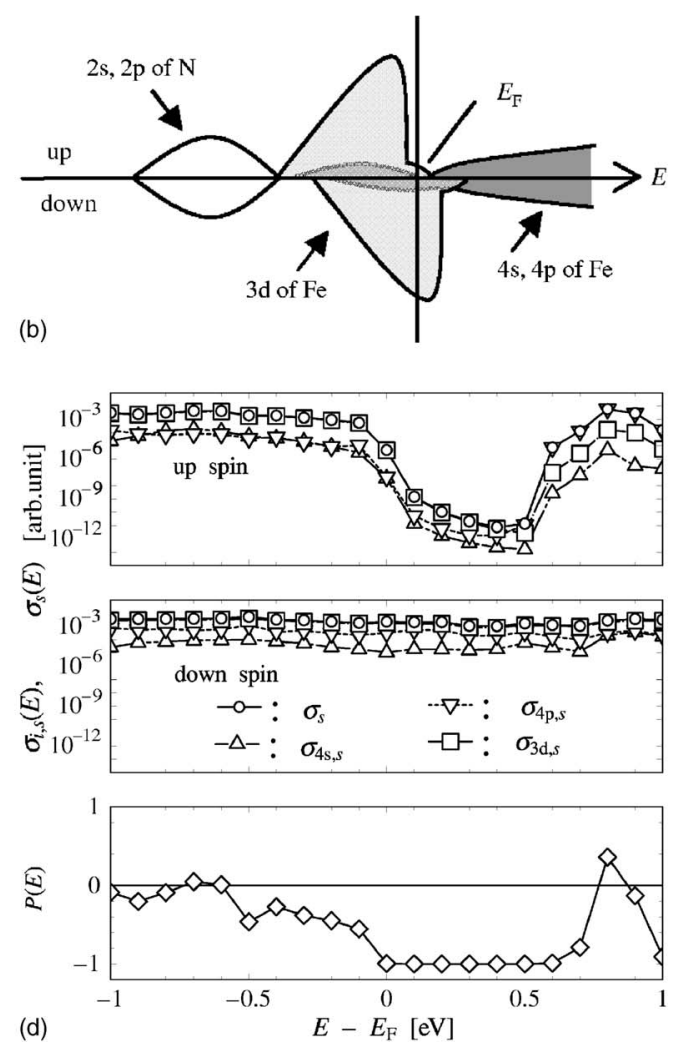

FIG. 1. Density of states, conductivities, and spin polarization ratios of $\mathrm{Fe}_{4} \mathrm{~N}$ : (a) $D_{s}(E)$ calculated using the FP calculation. (b) Schematic illustration of partial DOSs. The $4 s-4 p$ components are partly covered by the $3 d$ ones (see darkish parts in $3 d$ components). (c) $D_{s}(E)$ and $P_{\mathrm{DOS}}(E)$ for $-1 \mathrm{eV} \leqslant E-E_{\mathrm{F}} \leqslant 1 \mathrm{eV}$ of the TB model and the FP calculation. (d) $\sigma_{i, s}(E), \sigma_{s}(E)$, and $P(E)$ for $-1 \mathrm{eV} \leqslant E-E_{\mathrm{F}} \leqslant 1 \mathrm{eV}$. Here, $\sigma_{2 \mathrm{~s}, s}(E)$ and $\sigma_{2 \mathrm{p}, s}(E)$ for $\mathrm{N}$ are not shown because of their small values. 

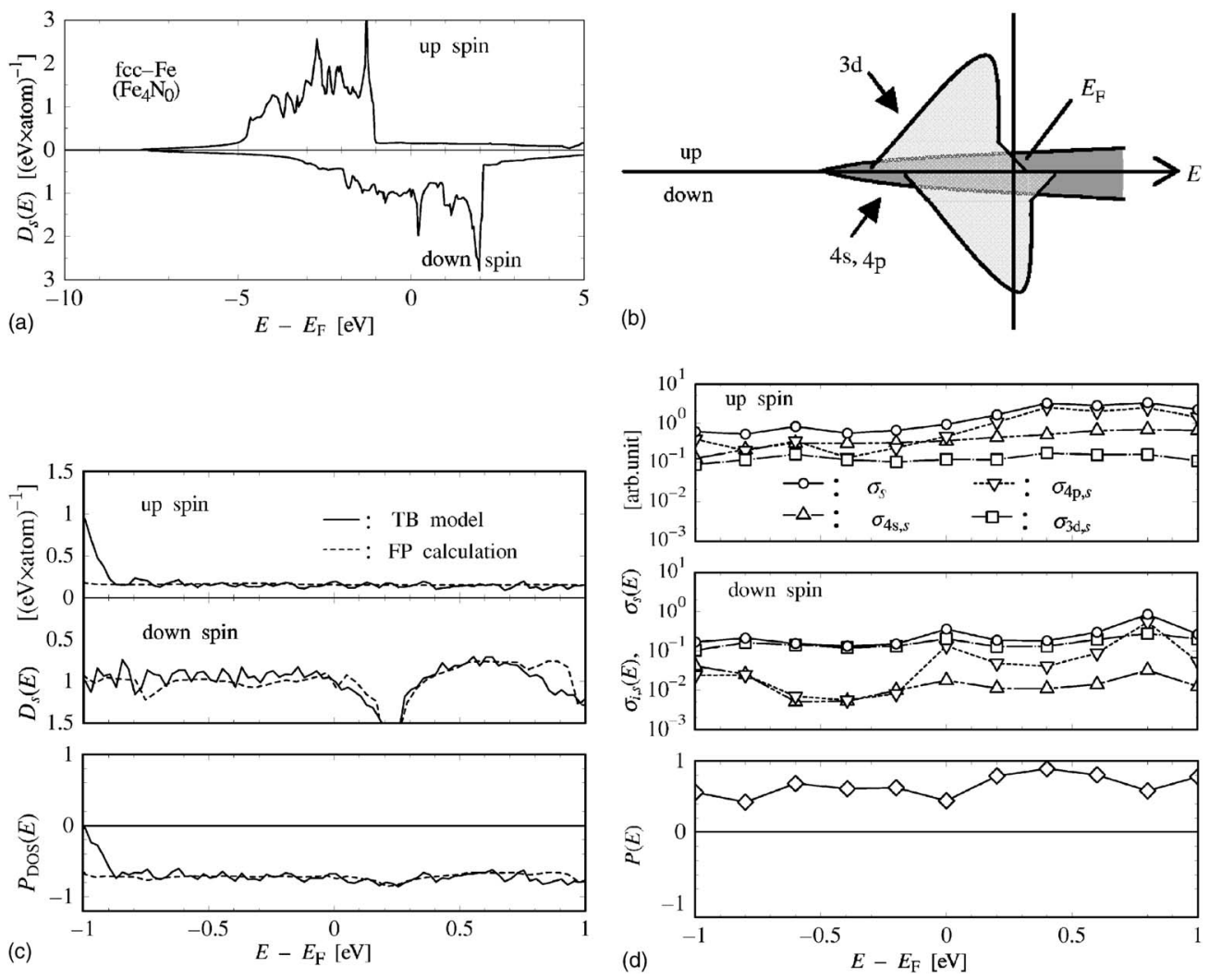

FIG. 2. The same as Fig. 1 for fcc-Fe $\left(\mathrm{Fe}_{4} \mathrm{~N}_{0}\right)$ in the ferromagnetic state with the equilibrium lattice constant of $\mathrm{Fe}_{4} \mathrm{~N}$ : $(\mathrm{a}) D_{s}(E)$ calculated using the FP calculation. (b) Schematic illustration of partial DOSs. The $4 s-4 p$ components are partly covered by the $3 d$ ones (see darkish parts in $3 d$ components). (c) $D_{s}(E)$ and $P_{\mathrm{DOS}}(E)$ for $-1 \mathrm{eV} \leqslant E-E_{\mathrm{F}} \leqslant 1 \mathrm{eV}$ of the TB model and the FP calculation. (d) $\sigma_{i, s}(E)$, $\sigma_{s}(E)$, and $P(E)$ for $-1 \mathrm{eV} \leqslant E-E_{\mathrm{F}} \leqslant 1 \mathrm{eV}$.

constant $D_{s}(E)$, and $P_{\mathrm{DOS}}(E)$ for $\mathrm{Fe}_{4} \mathrm{~N}$ using the FP calculation. The equilibrium lattice constant is evaluated as $3.810 \AA$, which has an error of less than $1 \%$ for an experimental value of $3.795 \AA .{ }^{12}$ As shown in Figs. 1(a) and 1(c), $D_{\downarrow}\left(E_{\mathrm{F}}\right)$ is higher than $D_{\uparrow}\left(E_{\mathrm{F}}\right)$. Partial DOSs (Ref. 14) schematically illustrated in Fig. 1(b) show that $3 d$ orbitals are dominant around $E_{\mathrm{F}}$, and $4 s$ and $4 p(4 s-4 p)$ orbitals are mainly located in an energy region higher than $E_{\mathrm{F}}$, while each orbital of $\mathrm{N}$ atoms mostly exists in an energy region lower than $E_{\mathrm{F}}$. Furthermore, $P_{\mathrm{DOS}}\left(E_{\mathrm{F}}\right)$ obtained by using $D_{s}\left(E_{\mathrm{F}}\right)$ of the FP calculation is evaluated to be -0.6 [see Fig. $1(\mathrm{c})]$.

Using the TB model with the parameters determined from the fitting of the dispersion curves, we obtain $D_{s}(E)$ and $P_{\mathrm{DOS}}(E)$ for $-1 \mathrm{eV} \leqslant E-E_{\mathrm{F}} \leqslant 1 \mathrm{eV}$. As seen from Fig. 1(c), $D_{s}\left(E_{\mathrm{F}}\right)$ and $P_{\mathrm{DOS}}\left(E_{\mathrm{F}}\right)$ of the TB model agree well with the respective ones of the $\mathrm{FP}$ calculation.

With the use of the TB model and the Kubo formula we calculate $\sigma_{i, s}(E), \quad \sigma_{s}(E)$, and $P(E)$ for $-1 \mathrm{eV} \leqslant E-E_{\mathrm{F}}$ $\leqslant 1 \mathrm{eV}$. The results are shown in Fig. 1(d). For $E \leqslant E_{\mathrm{F}}$, $\sigma_{3 \mathrm{~d}, \uparrow}(E)$ becomes relatively large owing to the high DOS of the $3 d$ orbitals of the up spin. For $E-E_{\mathrm{F}} \geqslant 0.6 \mathrm{eV}$, the $4 s$ $-4 p$ orbitals of the up spin contribute strongly to $\sigma_{\uparrow}(E)$ in spite of their low DOSs because their orbitals have large velocities. For $0 \mathrm{eV}<E-E_{\mathrm{F}}<0.6 \mathrm{eV}$, each $\sigma_{i, \uparrow}(E)$ has a pronounced valley reflecting the low DOS of the up spin. In this energy region, although the DOS of the up spin is actu- ally lower than that of the FP calculation, the qualitative behavior of $\sigma_{i, \uparrow}(E)$ appears to be valid because the DOS of the FP calculation has very few components of the $4 s-4 p$ orbitals and it is low. On the other hand, each $\sigma_{i, \downarrow}(E)$ is almost flat, and the $3 d$ orbitals of the down spin contribute largely to $\sigma_{\downarrow}(E)$ because of their high DOS. The total conductivity at $E_{\mathrm{F}}$ of the down spin $\sigma_{\downarrow}\left(E_{\mathrm{F}}\right)$ is much larger than $\sigma_{\uparrow}\left(E_{\mathrm{F}}\right)$. The SP ratio $P\left(E_{\mathrm{F}}\right)$ therefore takes almost -1.0 , indicating perfectly spin-polarized transport. The magnitude of the SP ratio $\left|P\left(E_{\mathrm{F}}\right)\right|$ is about 5.0 times as large as that of bcc-Fe.

In order to clarify the effect of an $\mathrm{N}$ atom on the transport, we investigated $D_{s}(E), P_{\mathrm{DOS}}(E), \sigma_{i, s}(E), \sigma_{s}(E)$, and $P(E)$ of fcc-Fe $\left(\mathrm{Fe}_{4} \mathrm{~N}_{0}\right)$ in the ferromagnetic state with an equilibrium lattice constant of $\mathrm{Fe}_{4} \mathrm{~N}$. Regarding $D_{s}(E)$ around $E_{\mathrm{F}}$ obtained using the FP calculation, $D_{\downarrow}(E)$ is much higher than $D_{\uparrow}(E)$ [see Figs. 2(a) and 2(c)]; for further details, low and broad DOSs of the $4 s-4 p$ orbitals of the up spin and a high DOS of the $3 d$ orbitals of the down spin are observed [see Fig. 2(b)]. The SP ratio of DOS, $P_{\mathrm{DOS}}\left(E_{\mathrm{F}}\right)$, is evaluated to be about -0.7 . Figure $2(\mathrm{c})$ also shows $D_{s}(E)$ and $P_{\mathrm{DOS}}(E)$ for $-1 \mathrm{eV} \leqslant E-E_{\mathrm{F}} \leqslant 1 \mathrm{eV}$; these values are obtained using the TB model with the determined parameters. It is found that $D_{s}\left(E_{\mathrm{F}}\right)$ and $P_{\mathrm{DOS}}\left(E_{\mathrm{F}}\right)$ of the TB model agree fairly well with the respective ones of the FP calculation. With the use of the TB model, we calculate $\sigma_{i, s}(E)$ and $\sigma_{s}(E)$ [see Fig. 2(d)]. In spite of the low DOSs of the $4 s-4 p$ orbitals of the up spin, 
their orbitals contribute strongly to $\sigma_{\uparrow}(E)$. This tendency originates from the large velocities of the $4 s-4 p$ orbitals. Moreover, $\sigma_{3 \mathrm{~d}, \downarrow}(E)$ is relatively large reflecting the high DOS of the $3 d$ orbitals of the down spin. The total conductivity at $E_{\mathrm{F}}$ of the up spin $\sigma_{\uparrow}\left(E_{\mathrm{F}}\right)$ then becomes larger than $\sigma_{\downarrow}\left(E_{\mathrm{F}}\right)$ even though $D_{\uparrow}\left(E_{\mathrm{F}}\right)$ is much lower than $D_{\downarrow}\left(E_{\mathrm{F}}\right)$. The SP ratio $P\left(E_{\mathrm{F}}\right)$ has a positive sign, opposite to the sign of $P_{\mathrm{DOS}}\left(E_{\mathrm{F}}\right)$. This sign of $P\left(E_{\mathrm{F}}\right)$ is also opposite to that of $\mathrm{Fe}_{4} \mathrm{~N}$. The magnitude of the SP ratio $\left|P\left(E_{\mathrm{F}}\right)\right|$ is evaluated to be 0.4 , which is between the $\left|P\left(E_{\mathrm{F}}\right)\right|$ of $\mathrm{Fe}_{4} \mathrm{~N}$ and that of bcc-Fe.

On the basis of the above investigations and the evaluated Slater-Koster parameters, we discuss the role of the $\mathrm{N}$ atom on the highly spin-polarized transport of $\mathrm{Fe}_{4} \mathrm{~N}$ using schematic illustrations of partial DOSs [see Figs. 1(b) and 2(b)]. Note that by merely introducing the nonmagnetic atom $\mathrm{N}$ to the body center position of fcc-Fe, $\left|P\left(E_{\mathrm{F}}\right)\right|$ becomes about 2.5 times as large as that of fcc-Fe and the sign of $P\left(E_{\mathrm{F}}\right)$ changes. In the present study, we find that, by adding $\mathrm{N}$ to fcc-Fe, $4 s-4 p$ bands of $\mathrm{Fe}$ are raised to a higher energy region by a large magnitude of transfer integrals between the $4 s-4 p$ orbitals of $\mathrm{Fe}$ and the $2 s$ and $2 p$ orbitals of $\mathrm{N}$, while $3 d$ bands do not change significantly owing to the small magnitude of the transfer integrals between the $3 d$ orbitals of Fe and the $2 s$ and $2 p$ orbitals of $\mathrm{N}$. These behaviors are explained by considering the bonding-antibonding states formed by the transfer integrals, which correspond to overlaps between both orbitals. A large portion of $4 s-4 p$ bands exists in an energy region higher than $3 d$ bands, and a small portion of them is located in the energy region of $3 d$ bands because of hybridizations with $3 d$ orbitals, and is then spinpolarized there. Hence, comparing $\mathrm{Fe}_{4} \mathrm{~N}$ with fcc-Fe, the proportion of $4 s-4 p$ bands is small in the vicinity of $E_{\mathrm{F}}$, and, in particular, the proportion of the up spin is extremely small; the $3 d$ bands of the down spin are dominant there. Therefore, the magnitude of the SP ratio $\left|P\left(E_{\mathrm{F}}\right)\right|$ increases, and $P\left(E_{\mathrm{F}}\right)$ becomes negative.

Finally, although it would be difficult to derive the general properties of phenomena from the present study alone, it seems possible that various ferromagnets consisting of magnetic elements and light elements have a large magnitude of SP ratios according to the same mechanism as that of $\mathrm{Fe}_{4} \mathrm{~N}$. In addition, when such ferromagnets are used as the FMEs, FME/insulator/FME junctions may exhibit large MR ratios, although the MR ratios are often influenced by the interfacial states and materials of the insulator. In fact, $\mathrm{CoFeB}$ electrodes bring about a very large $\mathrm{MR}$ effect in $\mathrm{CoFeB} / \mathrm{MgO} / \mathrm{CoFeB}$ junctions. ${ }^{5,6}$

In conclusion, $\left|P\left(E_{\mathrm{F}}\right)\right|$ of $\mathrm{Fe}_{4} \mathrm{~N}$ was evaluated to be almost 1.0, which was about 5.0 times as large as that of bcc-Fe and about 2.5 times as large as that of fcc-Fe. In comparison with fcc-Fe, it was shown that the large magnitude of the SP ratio originated from the contribution to the transport of $3 d$ bands, which was enhanced by introducing $\mathrm{N}$. We anticipate that $\mathrm{Fe}_{4} \mathrm{~N}$ will become an electrode with a high efficiency of spin injection. Furthermore, various ferromagnets consisting of magnetic elements and light elements may exhibit highly spin-polarized transport due to the present mechanism.

The authors thank T. Hoshino of Shizuoka University for useful discussions. One of the authors (S.K.) also thanks members of the nanomaterials theory group, AIST, for valuable discussions. This work has been supported by a competitive Grant program 2005 of Shizuoka University.
*Electronic address: tskokad@ipc.shizuoka.ac.jp

${ }^{1}$ K. Inomata, S. Okamura, R. Goto, and N. Tezuka, Jpn. J. Appl. Phys., Part 2 42, L419 (2003).

${ }^{2}$ Y. Sakuraba, J. Nakata, M. Oogane, H. Kubota, Y. Ando, A. Sakuma, and T. Miyazaki, Jpn. J. Appl. Phys., Part 2 44, L1100 (2005).

${ }^{3}$ S. Yuasa, A. Fukushima, T. Nagahama, K. Ando, and Y. Suzuki, Jpn. J. Appl. Phys., Part 2 43, L588 (2004).

${ }^{4}$ S. Yuasa, T. Nagahama, A. Fukushima, Y. Suzuki, and K. Ando, Nat. Mater. 3, 868 (2004).

${ }^{5}$ J. Hayakawa, S. Ikeda, F. Matsukura, H. Takahashi, and H. Ohno, Jpn. J. Appl. Phys., Part 2 44, L587 (2005).

${ }^{6}$ S. Ikeda, J. Hayakawa, Y. M. Lee, R. Sasaki, T. Meguro, F. Matsukura, and H. Ohno, Jpn. J. Appl. Phys., Part 2 44, L1442 (2005).

${ }^{7}$ S. Kokado and K. Harigaya, Phys. Rev. B 69, 132402 (2004).

${ }^{8}$ By performing the first principles calculation with the use of Vienna $a b$ initio Simulation Package code, we evaluated $\left|P_{\mathrm{DOS}}\left(E_{\mathrm{F}}\right)\right|$ of bcc-Fe to be about 0.3 .

${ }^{9}$ T. Hoshino, M. Asato, T. Nakamura, R. Zeller, and P. H. Dederichs, J. Magn. Magn. Mater. 272-276, e229 (2004).

${ }^{10}$ K. H. Jack, Proc. R. Soc. London, Ser. A 195, 34 (1948).
${ }^{11}$ G. Shirane, W. J. Takei, and S. L. Ruby, Phys. Rev. 126, 49 (1962).

${ }^{12}$ S. Nagakura, J. Phys. Soc. Jpn. 25, 488 (1968).

${ }^{13}$ W. Zhou, L. J. Qu, Q. M. Zhang, and D. S. Wang, Phys. Rev. B 40, 6393 (1989).

${ }^{14}$ A. Sakuma, J. Phys. Soc. Jpn. 60, 2007 (1991); J. Magn. Magn. Mater. 102, 127 (1991).

${ }^{15}$ S. Ishida and K. Kitawatase, J. Magn. Magn. Mater. 104-107, 1933 (1992).

${ }^{16}$ G. Kresse and J. Hafner, Phys. Rev. B 47, R558 (1993); 49, 14251 (1994); G. Kresse and J. Furthmuller, Comput. Mater. Sci. 6, 15 (1996); Phys. Rev. B 54, 11169 (1996).

${ }^{17}$ J. C. Slater and G. F. Koster, Phys. Rev. 94, 1498 (1954).

${ }^{18}$ R. Kubo, J. Phys. Soc. Jpn. 12, 570 (1957).

${ }^{19}$ J. P. Perdew, J. A. Chevary, S. H. Vosko, K. A. Jackson, M. R. Pederson, D. J. Singh, and C. Fiolhais, Phys. Rev. B 46, 6671 (1992).

${ }^{20}$ H. J. Monkhorst and J. D. Pack, Phys. Rev. B 13, 5188 (1976).

${ }^{21}$ E. Yu. Tsymbal and D. G. Pettifor, Phys. Rev. B 54, 15314 (1996).

${ }^{22}$ C. Kittel, Introduction to Solid State Physics, 6th ed. (Wiley, New York, 1986), p. 23. 